Linköping Studies in Science and Technology

Dissertations No. 1737

\title{
New Phenomena in the World of Peaked Solitons
} Marcus Kardell

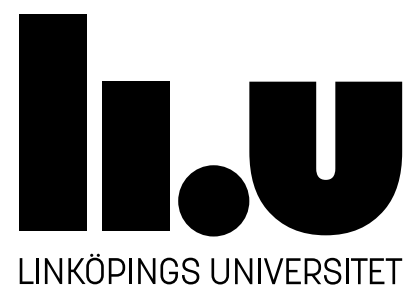

Department of Mathematics, Division of Mathematics and Applied Mathematics Linköping University, SE-581 83 Linköping, Sweden

Linköping 2016 


\section{New Phenomena in the World of Peaked Solitons}

Marcus Kardell

Linköping Studies in Science and Technology. Dissertations No. 1737.

ISSN 0345-7524

ISBN 978-91-7685-843-1

Copyright (@2016 Marcus Kardell, unless otherwise noted

Printed by LiU-Tryck, Linköping, Sweden, 2016 


\section{Abstract}

The aim of this work is to present new contributions to the theory of peaked solitons. The thesis consists of two papers, which are named "New solutions with peakon creation in the Camassa-Holm and Novikov equations" and "Peakon-antipeakon solutions of the Novikov equation" respectively.

In Paper I, a new kind of peakon-like solution to the Novikov equation is discovered, by transforming the one-peakon solution via a Lie symmetry transformation. This new kind of solution is unbounded as $x \rightarrow+\infty$ and/or $x \rightarrow-\infty$, and has a peak, though only for some interval of time. Thus, the solutions exhibit creation and/or destruction of peaks. We make sure that the peakon-like function is still a solution in the weak sense for those times where the function is non-differentiable. We find that similar solutions, with peaks living only for some interval in time, are valid weak solutions to the CamassaHolm equation, though it appears that these can not be obtained via a symmetry transformation.

In Paper II we investigate multipeakon solutions of the Novikov equation, in particular interactions between peakons with positive amplitude and antipeakons with negative amplitude. The solutions are given by explicit formulas, which makes it possible to analyze them in great detail. As in the Camassa-Holm case, the slope of the wave develops a singularity when a peakon collides with an antipeakon, while the wave itself remains continuous and can be continued past the collision to provide a global weak solution. However, the Novikov equation differs in several interesting ways from other peakon equations, especially regarding asymptotics for large times. For example, peakons and antipeakons both travel to the right, making it possible for several peakons and antipeakons to travel together with the same speed and collide infinitely many times. Such clusters may exhibit very intricate periodic or quasi-periodic interactions. It is also possible for peakons to have the same asymptotic velocity but separate at a logarithmic rate; this phenomenon is associated with coinciding eigenvalues in the spectral problem coming from the Lax pair, and requires nontrivial modifications to the previously known solution formulas which assume that all eigenvalues are simple. 


\section{Populärvetenskaplig sammanfattning}

Inom vågteori studeras så kallade solitoner, vilka kan beskrivas som vågpaket som rör sig med konstant form och hastighet. Typiska egenskaper är att utbredningen i rummet är begränsad, samt att två solitoner som kolliderar kan passera genom varandra utan att ändra form.

Fenomenet beskrevs redan 1834 av John Scott Russell, som ridande längs en kanal följde en "rundad, slät, väldefinierad upphöjning av vatten, vilken fortsatte sin bana längs kanalen synbarligen utan att ändra form eller förlora fart". Dåvarande våglära kunde inte förklara uppkomsten av sådana vågor, men moderna hydrodynamiska teorier innehåller ett antal modeller där solitoner är ett naturligt koncept.

I denna avhandling studeras vågekvationer som tillåter en särskild typ av spetsiga solitoner, så kallade peakoner (från engelskans "peaked soliton"). Avhandlingen utgörs av två artiklar som på olika sätt utvidgar förståelsen av detta fenomen.

I Artikel 1 beskrivs en ny typ av peakon-liknande våg där den spetsiga vågtoppen endast existerar under ett visst tidsintervall. Denna typ av våg kan visas förekomma i flera moderna vågekvationer, såsom Camassa-Holm-ekvationen och Novikovs ekvation.

I Artikel 2 studeras, i fallet med Novikovs ekvation, samspelet mellan peakoner och så kallade antipeakoner, vilket är vågor med spetsig vågdal istället för vågtopp. Artikeln beskriver vad som händer då peakoner kolliderar med antipeakoner, både i allmänhet och i några specialfall. 


\section{Acknowledgements}

Five years is quite a long time, but it does seem shorter when you have nice people around you. This is my attempt at thanking all those that helped me write the thesis, or supported me in other ways during the journey.

- My supervisor Hans Lundmark, for being a constant source of inspiration and help, whenever I needed it. His patience and friendliness is something every PhD student should have a right to find in their supervisor.

- My co-supervisors Stefan Rauch and Joakim Arnlind, for reading long manuscripts and giving valuable feedback at a moment's notice.

- Jacek Szmigielski, for the invitation to Canada and great hospitality during my visit.

- Other colleagues and fellow PhD students at the department, for making this a nice place to work at.

- Among them, in particular, John Karlsson, for a great friendship and interesting lunch discussions on just about every subject, ranging from the trickiest mathematics to the silliest nonsense.

- My family, who told me not to give up when I needed to hear it.

And the biggest and most special thank you goes to my fiancée Lotta, who always believed in me and supported me, every year and every day. It is finally done! 


\section{Contents}

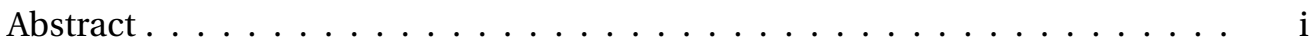

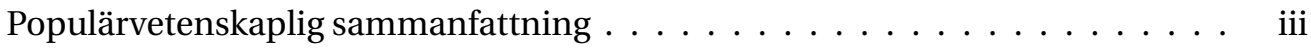

Acknowledgements . . . . . . . . . . . . . . . . v

Introduction 1

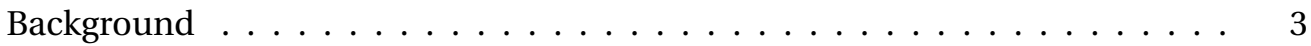

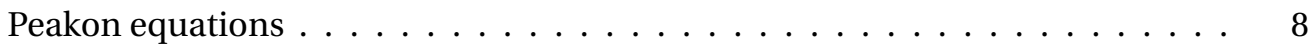

Paper I: New solutions with peakon creation in the Camassa-Holm and Novikov equations $\quad 15$

$\begin{array}{lll}1 & \text { Introduction } & 17\end{array}$

2 Novikov Solutions with Peakon Creation $\quad 18$

3 Peakon Creation in Related Equations 22

3.1 Camassa-Holm solutions with peakon creation . . . . . . . . . . 22

3.2 Degasperis-Procesi solutions with peakon creation? . . . . . . . . . 25

$\begin{array}{ll}\text { A Lie Symmetries } & \mathbf{2 7}\end{array}$

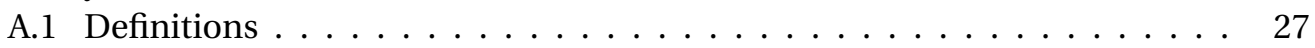

A.2 Using Jets . . . . . . . . . . . . . . . . . . . . . . . . 29

Paper II: Peakon-antipeakon solutions of the Novikov equation 35

$\begin{array}{lll}1 & \text { Introduction } & 37\end{array}$

2 Preliminaries 42

2.1 Weak solutions of the Novikov equation . . . . . . . . . . . . . 42

2.2 Comparison with $\mathrm{CH}$ and DP peakon-antipeakon collisions . . . . . . 45

2.3 Explicit solution formulas for Novikov multipeakons . . . . . . . . . . 47

3 Continuity of solutions at peakon-antipeakon collisions 53

4 Solutions of the Novikov equation with one peakon and one antipeakon 55

4.1 The governing ODEs . . . . . . . . . . . . . . . . 56

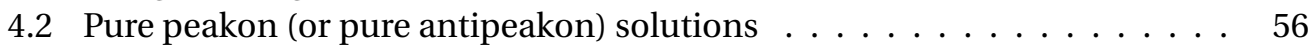

4.3 Extending the solution formulas to describing peakon-antipeakon solutions 61

4.4 Classification in terms of initial data . . . . . . . . . . . . . . . 63

4.5 Dynamics of a peakon-antipeakon pair in the case of positive simple eigenvalues . . . . . . . . . . . . . . . . . . . 66

4.6 Dynamics in the case of non-real eigenvalues $\ldots \ldots \ldots \ldots \ldots$ 
4.7 Dynamics in the case of a positive double eigenvalue . . . . . . . . . . 77

5 Peakon-antipeakon solutions with arbitrary $n$ and only simple eigenvalues 82

5.1 Spectral data and formulas for peakon-antipeakon solutions . . . . . . 83

5.2 More about collisions . . . . . . . . . . . . . . . 90

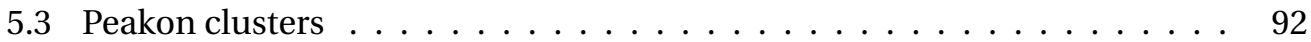

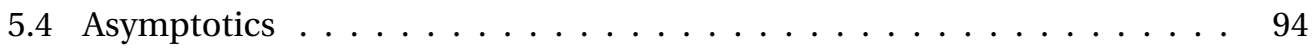

6 Peakon-antipeakon solutions with arbitrary $n$ and eigenvalues of any multi$\begin{array}{ll}\text { plicity } & 100\end{array}$

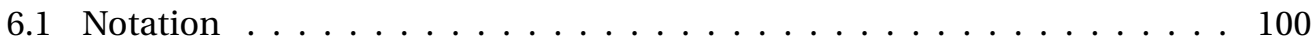

6.2 Multipeakon solution formulas . . . . . . . . . . . . . . 107

6.3 Example revisited: $n=2$, one double eigenvalue . . . . . . . . . 111

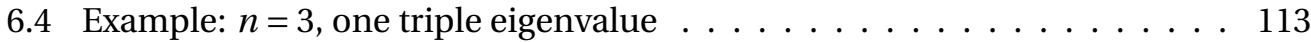

6.5 Example: $n=4$, two double eigenvalues . . . . . . . . . . . 118

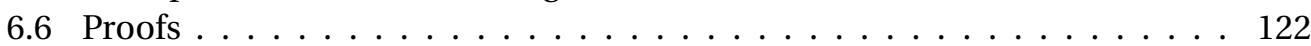

$\begin{array}{lll}7 & \text { Acknowledgements } & 133\end{array}$ 


\section{Introduction}




\section{Introduction}

This thesis presents several new contributions to the theory of peaked soliton solutions, so called peakons, in a number of different partial differential equations. The results are presented in two separate articles. Paper I is named "New solutions with peakon creation in the Camassa-Holm and Novikov equations", and is published in Journal of Nonlinear Mathematical Physics, 2015, 22(1). Paper II is coauthored with Hans Lundmark, and is named "Peakon-antipeakon solutions of the Novikov equation".

We begin the study of soliton equations by looking at the the KdV equation, which is the archetypical example of such equations. This gives us a chance to comment on some of the terminology used in the thesis. Then the relevant equations are introduced together with the concept of peakons, which are solutions to these equations in a certain weak sense.

\section{Background}

Before we get into the equations used in the papers, let us study the Korteweg-deVries $(\mathrm{KdV})$ equation

$$
u_{t}+u_{x x x}+6 u u_{x}=0
$$

where $u=u(x, t)$ is a function of space and time, and subscripts denote partial derivatives. This equation was introduced in the 19th century as a mathematical model for shallow water waves, and provides a nice example of features that we will see later in other equations, such as soliton solutions, integrability and inverse scattering. The constant in front of the term $u u_{x}$ has no particular relevance, as it can be changed to any other constant by a scaling transformation

$$
u=\frac{k}{6} v
$$

so that $v$ solves the equation

$$
v_{t}+v_{x x x}+k v v_{x}=0
$$

if and only if $u$ solves equation (1). In the literature, a number of different choices of $k$ are used, though we will stick to $k=6$, following Strauss's book [25].

\section{Solitons}

By soliton solutions, we mean localized traveling wave solutions, that interact with each other in a certain stable fashion. To find soliton solutions to the KdV equation, one makes the travelling wave ansatz

$$
u(x, t)=f(x-c t) .
$$

and replaces the partial derivatives $u_{x}=f^{\prime}, u_{t}=-c f^{\prime}$ into equation (1) to get the ordinary differential equation

$$
-c f^{\prime}+f^{\prime \prime \prime}+6 f f^{\prime}=0 \text {. }
$$


This equation is easily integrated to

$$
-c f+f^{\prime \prime}+3 f^{2}=C, \quad C \in \mathbb{R} .
$$

Multiplying by $2 f^{\prime}$ and integrating again gives

$$
-c f^{2}+\left(f^{\prime}\right)^{2}+2 f^{3}=C f+D, \quad C, D \in \mathbb{R} .
$$

We require that $f(x)$ and its derivatives tend to zero as $x \rightarrow \pm \infty$, since we are searching for localized waves. We thus put $C=D=0$, and solve the ODE

$$
\left(f^{\prime}\right)^{2}=c f^{2}-2 f^{3} .
$$

Besides the trivial solution $f(x)=0$, solutions are found by separating the equation into

$$
\int \frac{d f}{f \sqrt{c-2 f}}=\int d x
$$

which gives

$$
\frac{1}{\sqrt{c}} \ln \left|\frac{\sqrt{c-2 f}-\sqrt{c}}{\sqrt{c-2 f}+\sqrt{c}}\right|=x-x_{0} .
$$

Solving for $f$, we find that the travelling wave shape of the $\mathrm{KdV}$ equation is

$$
f(x-c t)=\frac{c}{2}\left(1-\left(\frac{1+e^{\sqrt{c}\left(x-x_{0}-c t\right)}}{1-e^{\sqrt{c}\left(x-x_{0}-c t\right)}}\right)^{2}\right)
$$

which we write as

$$
f(x-c t)=\frac{c}{2} \cosh ^{-2}\left(\frac{\sqrt{c}}{2}\left(x-x_{0}-c t\right)\right) .
$$

Note that this is a whole family of solutions, parametrized by the constants $c$ and $x_{0}$. For each choice of parameters one gets a soliton of fixed shape, where $x_{0}$ is the center of the wave profile at $t=0$, the amplitude is $\frac{c}{2}$ and the speed is equal to $c$. We thus see that taller solitons move faster than shorter ones. See Figure 1 for an example of a KdV soliton.

Now, consider a setup with a linear combination of two such KdV solitons, separated by a large distance at time $t=0$, and let their amplitudes be chosen such that the left one is taller. Note that the $\mathrm{KdV}$ equation can be interpreted as an evolution equation

$$
u_{t}=-u_{x x x}-6 u u_{x} .
$$

Thus, given the initial wave profile $u(x, 0)$, there should exist a well defined wave function $u(x, t)$, at least up to some time $t>0$. Initially, the separation of the solitons and their exponential decay means that they will not interact much. In fact, if one writes the linear combination as $u=v+w$, it follows that the product

$$
u u_{x}=v v_{x}+v w_{x}+w v_{x}+w w_{x}
$$




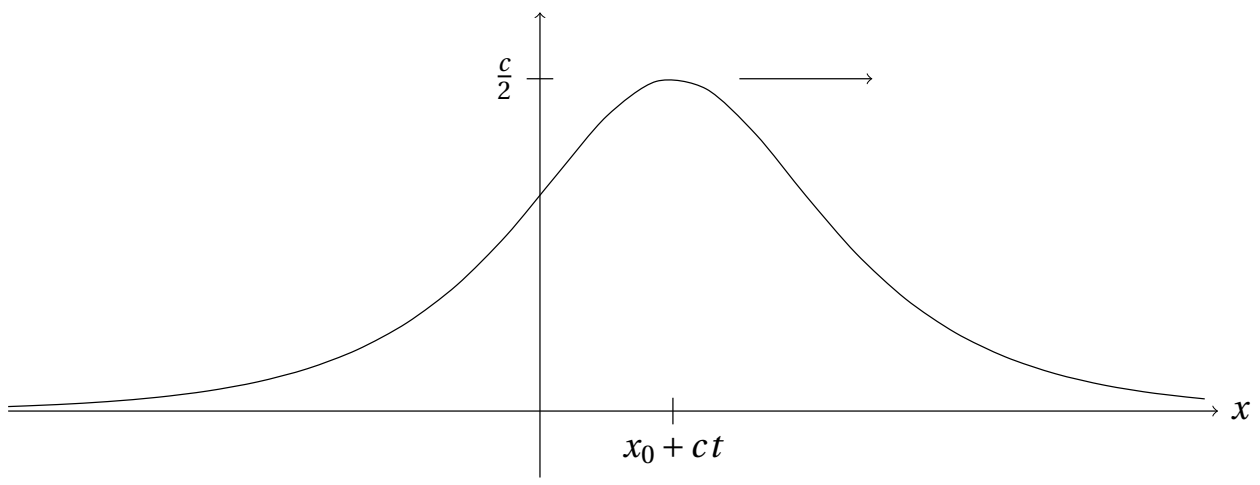

Figure 1: KdV soliton.

is initially approximately equal to $v v_{x}+w w_{x}$, since either $v$ or $w_{x}$ has to be close to zero for any given $x$, and the same holds for $v_{x}$ and $w$. Thus, we expect the solitons to first move along relatively unperturbed, according to their respective speeds. But what happens when the left (faster) soliton catches up with the right one?

Running numerical simulations with such setups, Kruskal and Zabusky [17] found that, after a complicated nonlinear interaction, the solitons emerge unscathed, so that there is a faster wave to the right of a slower one as $t \rightarrow+\infty$, with initial velocities and shapes preserved. In fact, one can show that this kind of behaviour translates to any linear combination of solitons, so that the number of solitons is preserved after interactions, with faster solitons ending up to the right of slower ones. It is this property of stability under interactions that characterizes localized traveling waves as solitons.

\section{Integrability}

The KdV equation turns out to be somewhat special among PDEs. The fact that one can find explicit solutions is not at all obvious just from looking at the equation - a small change in the order of derivatives in one of the terms might destroy the possibility of finding soliton solutions. This indicates that there is some property of the KdV equation which makes it particularly easy to work with, and this property is what we will call integrability.

In what follows we will simply think of a PDE as integrable if it has an infinite number of constants of motions and we can find explicit solutions to it. Note though, that there are a number of different ways of defining integrability, depending on the context. See [27] for various takes on the term.

We now want to study the constants of motion for the KdV equation. The first few can be found by trying to write the equation in the form

$$
\frac{\partial}{\partial t} P(u)+\frac{\partial}{\partial x} Q(u)=0 .
$$


In fact, if one is able to find such a relation, then (provided the integrals converge)

$$
\frac{\partial}{\partial t} \int_{-\infty}^{\infty} P(u) d x=-\frac{\partial}{\partial x} \int_{-\infty}^{\infty} Q(u) d x=0 .
$$

It is easy to see that the KdV equation (1) can be written in this form, with $P(u)=u$, $Q(u)=u_{x x}+3 u^{2}$, which means that

$$
\int_{-\infty}^{\infty} u d x
$$

is a constant of motion. Furthermore, multiplying the $\mathrm{KdV}$ equation by $2 u$ gives

$$
2 u u_{t}+2 u u_{x x x}+12 u^{2} u_{x}=0
$$

which is equivalent to

$$
\frac{\partial}{\partial t}\left(u^{2}\right)+\frac{\partial}{\partial x}\left(2 u u_{x x}-u_{x}^{2}+4 u^{3}\right)=0,
$$

so there is another constant of motion

$$
\int_{-\infty}^{\infty} u^{2} d x
$$

The next expression,

$$
\int_{-\infty}^{\infty}\left(\frac{u_{x}^{2}}{2}-u^{3}\right) d x
$$

is a bit more tricky to find, although one can easily check that its time derivative is equal to zero. The physical interpretations of these three constants are conservation of mass, momentum and energy, respectively.

The fact that KdV has infinitely many constants of motion is due to Miura, Gardner and Kruskal [23]. Define recursively a sequence $P_{i}(u)$ via

$$
\left\{\begin{array}{l}
P_{1}=u, \\
P_{i}=-\frac{\partial}{\partial x} P_{i-1}+\sum_{k=1}^{i-1} P_{k} P_{i-k}, \quad i \geq 2 .
\end{array}\right.
$$

Studying this sequence, one can show that

$$
\frac{\partial}{\partial t} \int_{-\infty}^{\infty} P_{i}(u) d x=0, \quad i \geq 1,
$$

which gives an infinite number of constants of motion. Note that the even-numbered ones are trivial, in the sense that

$$
P_{2 j}=\frac{\partial}{\partial x} Q_{j}
$$

for some functions $Q_{j}(u)$ that tend to zero as $x \rightarrow \pm \infty$. Thus the even-numbered expressions only give the trivial constant of motion, equal to zero. The odd-numbered $P_{i}$ will also contain some (but not all) terms that are integrated to zero. Removing those superfluous terms in $P_{1}, P_{3}$ and $P_{5}$ will lead to the three constants of motion found above. 


\section{Inverse scattering and Lax pairs}

In their study of the KdV equation [10], Gardner, Greene, Kruskal and Miura found a method for transforming a PDE to the region of spectral (or scattering) data, where time evolution is trivial, which actually gives a method for solving the equation given initial conditions

$$
u(x, 0)=\Phi_{0}(x) .
$$

Lax [18] reformulated the problem as a condition of compatibility for two linear PDEs. Consider the system

$$
\left\{\begin{array}{l}
L \Phi=\lambda \Phi \\
\Phi_{t}=B \Phi
\end{array}\right.
$$

where $L$ and $B$ are some given differential operators, and $\lambda$ is a fixed parameter. For this system to have solutions $\Phi$, it is necessary that the time derivative of the first equation is consistent with the second equation. By that, we mean that the derivative

$$
\frac{\partial}{\partial t}(L \Phi)=L_{t} \Phi+L \Phi_{t}=\lambda \Phi_{t}
$$

is consistent with the second equation, which is the case only if

$$
L_{t} \Phi+L B \Phi=B L \Phi .
$$

This equation can be conveniently written as an operator equation

$$
L_{t}=[B, L]
$$

using the usual commutator notation. What Lax did was to find a pair of operators $L$ and $B$, depending on a function $u$, such that the consistency condition (3) is equivalent to the KdV equation. In fact, with

$$
\begin{aligned}
& L=-\partial_{x}^{2}-u, \\
& B=-4 \partial_{x}^{3}-3 u \partial_{x}-3 \partial_{x} u,
\end{aligned}
$$

one gets that $L_{t}$ is just $-u_{t}$, while $[B, L] \Phi$ is equal to

$$
\left(-4 \partial_{x}^{3}-3 u \partial_{x}-3 \partial_{x} u\right)\left(-\Phi_{x x}-u\right)-\left(-\partial_{x}^{2}-u\right)\left(-4 \Phi_{x x x}-3 u_{x} \Phi-6 u \Phi_{x}\right),
$$

which after cancellation yields the expression

$$
6 u u_{x} \Phi+u_{x x x} \Phi .
$$

Thus, the Lax equation with this choice for the operators $B$ and $L$ is equivalent to the KdV equation, as desired.

Let us now look at the problem from the other direction. Given any function $u$ that solves the KdV equation, try to solve the eigenvalue problem

$$
L \Phi=\lambda \Phi,
$$


where one requires $\Phi(x)$ to be a nonzero function such that

$$
\int|\Phi| d x<\infty
$$

With the operator $L=-\partial_{x}^{2}-u$ taken from Lax's pair, this equation is known as the timeindependent Schrödinger equation of quantum mechanics, and gives rise to a discrete spectrum of eigenvalues (see [25])

$$
\lambda_{N} \leq \lambda_{N-1} \leq \cdots \leq \lambda_{1}<0,
$$

with corresponding eigenfunctions $\Phi_{n}(x, t)$. The limiting behaviour of these eigenfunctions as $x \rightarrow \infty$ is

$$
\Phi_{n}(x, t) \sim c_{n}(t) e^{-\sqrt{-\lambda_{n}} x},
$$

where the time dependence (which can be found via the second equation of the Lax system) is simply given by

$$
c_{n}(t)=c_{n}(0) e^{4\left(-\lambda_{n}\right)^{\frac{3}{2}} t} .
$$

We can now describe (in somewhat vague terms) the steps that constitute the inverse scattering method for computing solutions to the KdV equation.

First, given $u(x, 0)=\Phi_{0}(x)$, compute the scattering data at time 0 . This data consists of the time independent spectrum $\left(\lambda_{n}\right)_{n=1}^{N}$, together with the parameters $\left(c_{n}(0)\right)_{n=1}^{N}$. Since the time dependence is known, one immediately finds the scattering data $\left(\lambda_{n}, c_{n}\right)$ at an arbitrary time $t>0$. The third step, which is the hardest one, is known as the inverse scattering problem, where one must find $u(x, t)$ given scattering data at arbitrary time $t$. (It turns out that one more scattering variable is needed to uniquely reconstruct the function $u$, but the details are beyond the scope of this presentation.)

In general, the same kind of methods can be applied to any PDE which has an associated Lax pair. The difficult part is to find the inverse mapping from the spectral region back to the original function, and sometimes also finding enough spectral data to guarantee uniqueness.

Inverse scattering techniques are of particular importance in Paper II, where we consider solution formulas obtained through inverse scattering for the Novikov equation in [13].

\section{Peakon equations}

In Paper I and II, three more recently discovered partial differential equations are studied, all somewhat similar to the KdV equation in that they are integrable and admit soliton solutions. One important difference though, is that the soliton solutions to these equations turn out to have non-smooth peaks, which gives rise to the term 'peakons'. In this section we give a short historical overview describing the partial differential equations of interest to us, and what is known about peakon solutions to these equations. 


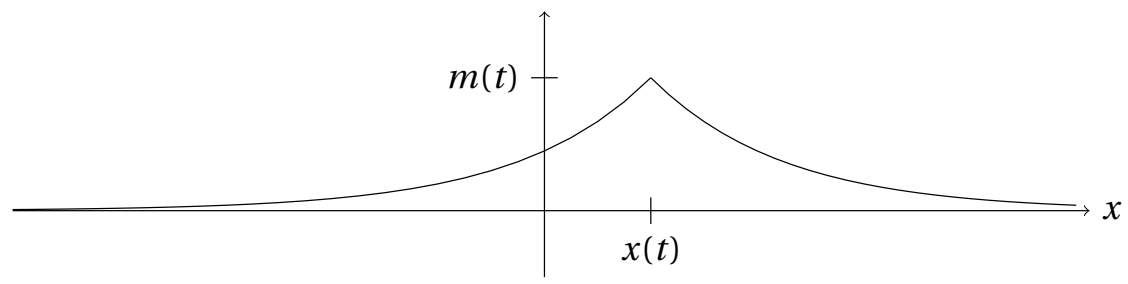

Figure 2: Peakon profile.

\section{The Camassa-Holm $b$-family}

The first object of interest is the following family of third order quadratically nonlinear PDEs,

$$
u_{t}-u_{x x t}=-(b+1) u u_{x}+b u_{x} u_{x x}+u u_{x x x}, \quad(x, t) \in \mathbb{R}^{2},
$$

where $b$ is a fixed parameter. Choosing $b=2$ gives the dispersionless version of the much studied Camassa-Holm (CH) equation [4] which was first developed in 1993 as a new model of shallow water waves. If one instead chooses $b=3$ in (4), it turns into the Degasperis-Procesi (DP) equation [9] from 1999.

Both equations are of interest in wave theory as they accomodate wave breaking, i.e., the slope of the wave profile may tend to infinity in finite time. It is interesting to note that the equations in the family are integrable only for $b=2$ and $b=3$, according to a number of integrability tests $[9,22,14,16]$.

Let us first study some properties of the Camassa-Holm equation, which appears as one of the main equations in Paper I. The original form of the equation includes a dispersion term,

$$
u_{t}-u_{x x t}+\kappa u_{x}=-3 u u_{x}+2 u_{x} u_{x x}+u u_{x x x},
$$

with the constant $\kappa$ related to the critical shallow water wave speed. Like for the KdV equation, soliton solutions can be shown to exist in this equation, but it is difficult to find explicit solution formulas. Camassa and Holm showed, that in the limit as $\kappa \rightarrow 0$, the solitons tend to to a very simple limiting shape, given by the function $e^{-|x|}$. Note though, that this shape is no longer smooth, as there is a peak where the left and right derivatives do not coincide. Camassa and Holm thus coined the term 'peakons', short for peaked solitons.

To study time dependent peakon solutions, consider the expression

$$
u(x, t)=m(t) e^{-|x-x(t)|}= \begin{cases}m(t) e^{-x+x(t)}, & x \geq x(t), \\ m(t) e^{x-x(t)}, & x \leq x(t) .\end{cases}
$$

See Figure 2 for a picture of the wave profile. If $m(t)<0$, the wave instead has a trough (downward pointing peak), and we call this an antipeakon.

One reason for the interest in peakon solutions is that they behave nicely under taking linear combinations, like we saw for the KdV solitons. Let us use the term multi- 


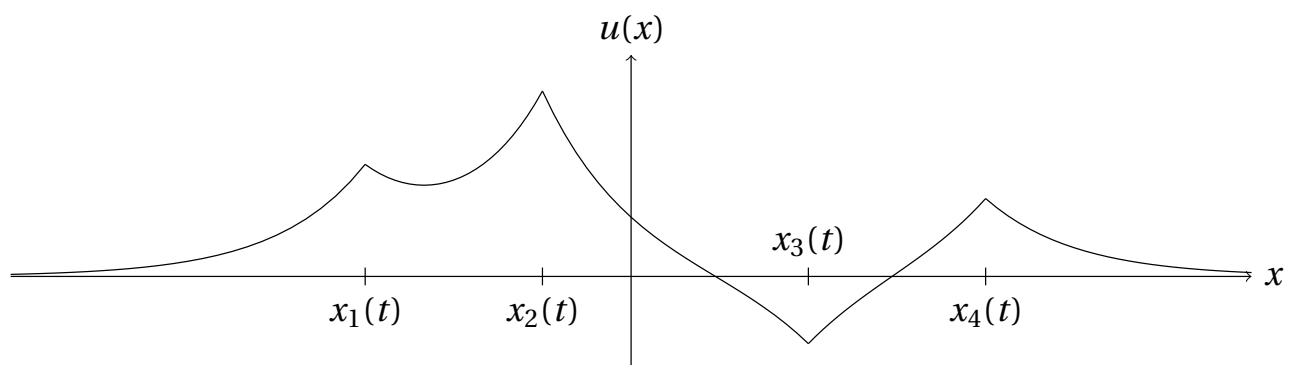

Figure 3: Multipeakon.

peakon to describe sums of peakons

$$
u(x, t)=\sum_{k=1}^{n} m_{k}(t) e^{-\left|x-x_{k}(t)\right|} .
$$

Sometimes one assumes $m_{k}>0$, which is the so called pure peakon case, whereas all $m_{k}<0$ corresponds to the pure antipeakon case. The remaining case, where not all $m_{k}$ have the same sign, we call the mixed (peakon/antipeakon) case. See Figure 3 for an example of a multipeakon wave profile for a given time $t$.

Note that multipeakons cannot be solutions to the partial differential equations (4) in a strong sense, since they are non-differentiable. The problem lies in multiplying $u_{x}$ with $u_{x x}$, since $u_{x x}$ contains Dirac deltas exactly at the jump discontinuities of $u_{x}$. To get around this, one rewrites the $b$-family as

$$
\left(1-\partial_{x}^{2}\right) u_{t}+\left(b+1-\partial_{x}^{2}\right) \partial_{x}\left(\frac{1}{2} u^{2}\right)+\partial_{x}\left(\frac{3-b}{2} u_{x}^{2}\right)=0 .
$$

A function $u(x, t)$ is said to be a weak solution if $u(\cdot, t) \in W_{\text {loc }}^{1,2}(\mathbb{R})$ for each fixed $t$, which means that $u(\cdot, t)^{2}$ and $u_{x}(\cdot, t)^{2}$ are locally integrable functions, if $u_{t}(\cdot, t)$ defined as the limit of a difference quotient exists as a distribution, and if (7) is satisfied for all $t$ in the distributional sense.

With the $n$-peakon ansatz (6), our PDEs are easily seen to be satisfied on the intervals where the multipeakon is differentiable, since each exponential function is a solution. Studying what goes on at the locations of each peak, one finds that the PDEs simplify into a system of $2 n$ ODEs in the variables $\left(x_{k}, m_{k}\right)$, which denote the position and height respectively of peakon $k$. For the $b$-family, this system is

$$
\left\{\begin{array}{l}
\dot{x}_{k}=\sum_{i=1}^{n} m_{i} e^{-\left|x_{k}-x_{i}\right|}, \\
\dot{m}_{k}=(b-1) m_{k} \sum_{i=1}^{n} m_{i} \operatorname{sgn}\left(x_{k}-x_{i}\right) e^{-\left|x_{k}-x_{i}\right|},
\end{array}\right.
$$

where we use the convention that sgn $0=0$.

For $n=1$, the system reduces to

$$
\left\{\begin{array}{l}
\dot{x}_{1}=m_{1}, \\
\dot{m}_{1}=0
\end{array}\right.
$$


which means that the peakon $u(x, t)=m_{1} e^{-\left|x-m_{1} t\right|}$ clearly is a travelling wave solution, maintaining its shape and height, travelling with constant speed equal to its height. Note that in this case, antipeakons move to the left while peakons move to the right.

For $n>1$, the interaction between peakons makes the ODE systems considerably more complicated. The system (8) was solved in the pure peakon sector using inverse scattering techniques for Camassa-Holm in [1], and the mixed case was solved in [2].

It turns out that in the pure peakon sector, there are no collisions amongst peakons, i.e., the coordinates $x_{1}(t)<x_{2}(t)<\cdots<x_{n}(t)$ remain separated for all times. This is not necessarily true in the mixed case, where collisions may occur, causing some $m_{k}$ to tend to infinity. Thus one has to be careful with the meaning of continuing a solution beyond a collision. In [12] it was shown how to obtain global multipeakon solutions of the Camassa-Holm equation, by introducing a new system of ODEs which is well-posed even at collisions. See also [3] for how to resolve singularities for more general kinds of solutions of the Camassa-Holm equation.

Finally, a word on the Degasperis-Procesi equation. Most of what we wrote about the Camassa-Holm equation carries through to the DP case. It is worth noting though that the situation is slightly different when it comes to weak solutions. Functions in $W_{\text {loc }}^{1, p}(\mathbb{R})$ are continuous by the Sobolev embedding theorem, but if one puts $b=3$ in (7), the term

$u_{x}^{2}$ disappears, so one only has to require that $u(\cdot, t) \in L_{\text {loc }}^{2}(\mathbb{R})$. Thus the DegasperisProcesi equation admits solutions that are not continuous, see for example $[19,5,6]$, while $\mathrm{CH}$ does not.

The DP peakon ODEs where solved in [20] in the pure peakon case, again with inverse scattering, and [26] dealt with the mixed peakon-antipeakon case.

\section{The Novikov equation}

Most prevalent in the thesis is the Novikov equation [24, 15]

$$
u_{t}-u_{x x t}=-4 u^{2} u_{x}+3 u u_{x} u_{x x}+u^{2} u_{x x x}
$$

which appears in both papers. This equation has a similar structure to $\mathrm{CH}$ and $\mathrm{DP}$, but instead has cubic nonlinearities in the right hand side.

We mention here also the Geng-Xue (GX) system [11]

$$
\left\{\begin{array}{l}
u_{x x t}-u_{t}=\left(u_{x}-u_{x x x}\right) u v+3\left(u-u_{x x}\right) v u_{x}, \\
v_{x x t}-v_{t}=\left(v_{x}-v_{x x x}\right) u v+3\left(v-v_{x x}\right) u v_{x},
\end{array}\right.
$$

which is studied briefly in the Appendix of Paper I. This system can be thought of as a two-component generalization of the Novikov equation, as the system reduces to two copies of (10) when replacing $u=v$.

To define weak solutions of the Novikov equation we write (10) as

$$
\left(1-\partial_{x}^{2}\right) u_{t}+\left(4-\partial_{x}^{2}\right) \partial_{x}\left(\frac{1}{3} u^{3}\right)+\partial_{x}\left(\frac{3}{2} u u_{x}^{2}\right)+\frac{1}{2} u_{x}^{3}=0 .
$$


We require that $u(\cdot, t) \in W_{\mathrm{loc}}^{1,3}(\mathbb{R})$ for all $t$, so that $u^{3}$ and $u_{x}^{3}$ are locally integrable. It then follows from Hölder's inequality with conjugate indices 3 and $\frac{3}{2}$ that the term $u u_{x}^{2}$ is locally integrable as well. We also require that the time derivative $u_{t}$ exists as a distribution defined as the limit of a difference quotient for almost every $t$. Then it makes sense to call $u$ a weak solution to the Novikov equation if the left hand side of (12) gives zero when acting on a test function, for all $t$ where $u_{t}$ exists.

The Novikov equation is of interest in this thesis, since it admits peakon solutions too. By using the multipeakon ansatz as before, a similar system to (8) can be constructed for peakon dynamics in the Novikov equation,

$$
\left\{\begin{array}{l}
\dot{x}_{k}=\left(\sum_{i=1}^{n} m_{i} e^{-\left|x_{k}-x_{i}\right|}\right)^{2}, \\
\dot{m}_{k}=m_{k}\left(\sum_{i=1}^{n} m_{i} e^{-\left|x_{k}-x_{i}\right|}\right)\left(\sum_{i=1}^{n} m_{i} \operatorname{sgn}\left(x_{k}-x_{i}\right) e^{-\left|x_{k}-x_{i}\right|}\right) .
\end{array}\right.
$$

Note that here, a single peakon travels with constant speed equal to the square of its height, which is different from the previous equations, in that both peakons and antipeakons move to the right in the Novikov equation. Pure multipeakon solutions to Novikov and GX were studied in [13] and [21] respectively, while the peakon-antipeakon interactions are the topic of Paper II.

\section{References}

[1] Beals R., Sattinger D., Szmigielski J., Multi-peakons and a theorem of Stieltjes, Inverse Problems, 15(1):L1-L4, 1999.

[2] Beals R., Sattinger D., Szmigielski J., Multipeakons and the classical moment problem, Advances in Mathematics, 154:229-257, 2000.

[3] Bressan A., Constantin A., Global conservative solutions of the Camassa-Holm equation, Arch. Ration. Mech. Anal., 183(2):215-239, 2007.

[4] Camassa R., Holm D., An integrable shallow water equation with peaked solitons, Phys. Rev. Lett., 71(11):1661-1664, 1993.

[5] Coclite G., Karlsen K., On the well-posedness of the Degasperis-Procesi equation, J. Funct. Anal., 233(1):60-91, 2006.

[6] Coclite G., Karlsen K., On the uniqueness of discontinuous solutions to the Degasperis-Procesi equation, J. Differential Equations, 234(1):142-160, 2007.

[7] Constantin A., Lannes D., The hydrodynamical relevance of the Camassa-Holm and Degasperis-Procesi equations, Archive for Rational Mechanics and Analysis, 192(1):165-186, 2009.

[8] Cotter C., Holm D., Ivanov R., Percival J., Waltzing peakons and compacton pairs in a cross-coupled Camassa-Holm equation, J. Phys. A: Math. Theor, 44(26):265205, 2011. 
[9] Degasperis A., Procesi M., Asymptotic integrability, Symmetry and perturbation theory (SPT 98, Rome), 23-37, 1999.

[10] Gardner C., Greene J., Kruskal M., Miura R., Method for solving the KortewegdeVries equation, Phys. Rev. Lett., 19(19):1095-1097, 1967.

[11] Geng X., Xue B., An extension of integrable peakon equations with cubic nonlinearity, Nonlinearity, 22(8):1847-1856, 2009.

[12] Holden H., Raynaud X., Global conservative multipeakon solutions of the Camassa-Holm equation, J. Hyperbolic Diff. Eq., 4(1):39-64, 2007.

[13] Hone A. N. W., Lundmark H., Szmigielski J., Explicit multipeakon solutions of Novikov's cubically nonlinear integrable Camassa-Holm type equation, Dynamics of Partial Differential Equations, 6(3):253-289, 2009, arXiv:0903.3663 [nlin.SI].

[14] Hone A., Wang J., Prolongation algebras and Hamiltonian operators for peakon equations, Inverse Problems, 19(1):129-145, 2003.

[15] Hone A., Wang J., Integrable peakon equations with cubic nonlinearity, J. Phys. A, 41(37):372002, 2008.

[16] Ivanov R., On the integrability of a class of nonlinear dispersive wave equations, $J$. Nonlinear Math. Phys., 12(4):462-468, 2005.

[17] Kruskal M. D., Zabusky, N. J., Interaction of "solitons" in a collisionless plasma and the recurrence of initial states, Phys. Rev. Lett., 15(6):240-243, 1965.

[18] Lax P., Integrals of nonlinear equations of evolution and solitary waves, Comm. Pure Applied Math, 21(5):467-490, 1968.

[19] Lundmark H., Formation and dynamics of shock waves in the Degasperis-Procesi equation, J. Nonl. Sci., 17(3):169-198, 2007.

[20] Lundmark H., Szmigielski J., Degasperis-Procesi peakons and the discrete cubic string, IMRP Int. Math. Res. Pap., 2005(2):53-116, 2005.

[21] Lundmark H., Szmigielski J., An inverse spectral problem related to the GengXue two-component peakon equation, arXiv:1304.0854 [nlin.SI], 2013. To appear in Mem. Amer. Math. Soc.

[22] Mikhailov A., Novikov V., Perturbative symmetry approach, J. Phys. A., 35(22):47754790, 2002.

[23] Miura R. M., Gardner C. S., Kruskal M. D., Korteweg-de Vries equation and generalizations. II. Existence of conservation laws and constants of motion, Journal of Mathematical Physics, 9(8):1204-1209, 1968. 
[24] Novikov V., Generalizations of the Camassa-Holm equation, J. Phys. A, 42(34):342002, 14, 2009.

[25] Strauss W. A., Partial Differential Equations - an introduction, Jon Wiley \& Sons, Inc, 2008.

[26] Szmigielski J., Zhou L., Peakon-antipeakon interactions in the Degasperis-Procesi Equation, In Algebraic and geometric aspects of integrable systems and random matrices, vol. 593 of Contemp. Math., pp. 83-107, Amer. Math. Soc., Providence, RI, 2013, arXiv:1301.0171 [math-ph].

[27] Zakharov V. E. (Ed.), What Is Integrability?, Springer-Verlag, 1991. 